\title{
e-Migrinter
}

16 | 2017

Récits d'exilés

\section{Ceuta, dernière frontière}

Témoignage à charge d'un migrant subsaharien illégalisé

\section{Frédérik Detue}

\section{(2) OpenEdition}

Journals

Édition électronique

URL : https://journals.openedition.org/e-migrinter/933

DOI : 10.4000/e-migrinter.933

ISSN : 1961-9685

Éditeur

UMR 7301 - Migrinter

Référence électronique

Frédérik Detue, «Ceuta, dernière frontière », e-Migrinter [En ligne], 16 | 2017, mis en ligne le, consulté le 21 septembre 2021. URL : http://journals.openedition.org/e-migrinter/933 ; DOI : https://doi.org/

10.4000/e-migrinter.933

Ce document a été généré automatiquement le 21 septembre 2021.

Tous droits réservés 


\title{
Ceuta, dernière frontière
}

\author{
Témoignage à charge d'un migrant subsaharien illégalisé
}

\author{
Frédérik Detue
}

"Sa propre bataille allait être encore très longue,
la suite obscure, et son passé était ténébreux. "
Fabien Didier Yene (Yene, 2010 : 95) ${ }^{1}$

L'étude du témoignage comme genre littéraire nous confronte constamment, et sans nous y habituer, à des récits qui, comme dit Jean Cayrol, ne dépouillent pas le réel de la «tunique brûlante dont il [est] enveloppé» (Cayrol, 1953: 576). Celles et ceux qui témoignent en littérature et qui prennent modèle sur la déposition en justice font un récit aussi sobre que possible du crime de masse dont ils sont, au moins temporairement, les rescapés. Mais le but qu'ils se donnent de nous faire imaginer les faits inimaginables qu'ils ont vus et vécus représente une bien redoutable épreuve, à la fois pour eux, qui revivent les faits en les décrivant, et pour nous, qui recueillons ces faits vécus en nous. Cette épreuve du témoignage, c'est, pour le dire en un mot, celle de la stupéfaction. Les témoins rescapés de crimes de masse sont des êtres stupéfaits des violences qu'ils ont subies dans leurs corps et leurs âmes et qui ne laissent pas de leur paraître à la fois invraisemblables et intolérables. Les meilleurs témoignages, dès lors, sont ceux qui font entendre cette protestation individuelle née au contact des faits, étant donné qu'elle seule conditionne une vraie compréhension. Or une chose est de ne pas se résigner à des faits criminels du passé, sachant que «[r]ien n'est résolu, [...] et [que] remettre en mémoire ne veut pas dire remiser dans la mémoire » (Améry, 2005 : 20 ) ; et une autre chose est de ne pas se résigner à des faits criminels qui continuent à se perpétrer quotidiennement au présent, quasiment sous nos yeux. Ce n'est qu'une affaire de degré dans l'urgence, sans doute, mais la « tunique brûlante » du réel n'en est que plus insupportable. Surtout, nous sentons bien que nous portons une responsabilité encore plus grande si nous détournons notre attention.

2 En l'occurrence, je me propose d'examiner un corpus testimonial relatif au sujet des routes migratoires vers l'Europe, dont personne n'ignore l'actualité. J'étudie spécialement, en le confrontant à d'autres sources documentaires, le témoignage Migrant au pied du mur de Fabien Didier Yene, édité en 2010. Les faits que l'auteur relate 
dans cet ouvrage s'étendent pour l'essentiel sur quatre ans, de 2003 à 2007. S'exilant du Cameroun en raison de «menaces [...] trop lourdes de conséquences" (Yene, $2010: 49$ ) et formant progressivement le projet de gagner l'Union européenne, Fabien Didier Yene n'a à aucun moment envisagé l'éventualité d'obtenir un visa Schengen, comme si toute tentative d'emprunter une voie sûre et régulière d'entrée et de séjour dans cet espace européen était par avance vouée à l'échec. Son expérience de migration est ainsi exemplaire de ces mobilités d'emblée "illégalisées " ou "irrégularisées " ${ }^{2}$ vécues par tous les Africains subsahariens qui tentent «l'aventure » (Yene, $2010: 87$ ). Il a emprunté une route migratoire qui l'a conduit, comme beaucoup de ses "frères " noirs d'Afrique de l'Ouest et du Centre, au nord du Maroc, aux abords des enclaves coloniales espagnoles de Ceuta et de Melilla. En 2003, les frontières espagnoles en Méditerranée occidentale passaient encore, parmi les frontières extérieures de l'Union européenne, pour les moins imperméables. En particulier, malgré leur blindage d'année en année plus sophistiqué, les frontières terrestres des deux enclaves paraissaient moins dangereuses que les frontières maritimes. Quatre ans durant, cependant, Fabien Didier Yene a survécu à des épreuves migratoires d'une telle violence qu'il a éprouvé le besoin d'en témoigner publiquement afin de les porter à la connaissance de tous. Il a même pour cela affronté "des conditions inimaginables ", étant donné qu'il était encore bloqué au Maroc, précisément «à Rabat» (Yene, 2010:258), et qu'il y était traité comme un étranger indésirable.

Le témoignage de Fabien Didier Yene est précieux par ce qui le motive si impérieusement. Il est d'abord une attestation en littérature dans laquelle l'auteur veut faire toute la lumière sur les violences politiques qu'il a vues et subies ; il entend, dit-il, " porter témoignage sur les horreurs vécues par les Subsahariens dans leur traversée du désert pour une Terre promise » (Yene, $2010: 259)$. Ces « horreurs » qui provoquent une mort de masse parmi les exilés sont un état d'exception d'autant plus intolérable qu'il perdure année après année sans que personne ne semble s'en émouvoir. Par sa prise de parole publique, l'auteur espère précisément briser le relatif silence entretenu au sujet de ces violences de façon à en finir avec l'impunité. Par délégation, au nom de ses «frères » et "sœurs » d'Afrique subsaharienne, il décrit ainsi les effets mortifères d'une partition du monde postcoloniale qui met au ban de l'humanité des femmes et des hommes en quête de liberté.

L'image du titre Migrant au pied du mur semble focaliser l'attention sur «la dernière étape à franchir» (Yene, 2010: 195) en vue d'atteindre l'Europe. Dans l'édition Atlantica-Séguier, c'est ce qu'étaie la publication, en marge du témoignage, de textes d'intervention tels que la Déclaration du « Sommet citoyen sur les migrations » de 2008 à Montreuil, intitulée « Des ponts, pas des murs » (Yene, $2010: 273-281$ ). Articulé ainsi à l'intense activité militante menée conjointement par l'auteur au Maroc ${ }^{4}$, le témoignage se présente en premier lieu comme une déposition contre les responsables européens de la "teichopolitique ", ou "politique de cloisonnement de l'espace " (Ballif, Rosière, 2009 : 194)5 $)^{5}$ matérialisée depuis 1993 dans les enclaves par les barrières frontalières. Cependant, ce n'est pas la moindre qualité de Migrant au pied du mur que de décrire l'expérience migratoire de son auteur pas à pas depuis son commencement; le récit de Fabien Didier Yene, qui atteste de violences vues et subies déjà bien avant d'atteindre le nord du Maroc, fait ainsi ressortir que la frontière est en réalité moins une ligne qu'un réseau ${ }^{6}$ et que la «teichopolitique » européenne n'est en ce sens que la partie la plus 
démonstrative du "dispositif migratoire " (Feldman, 2011) mis en place pour stopper les mouvements de migration.

\section{Témoignage de migration et représentations}

Il est instructif pour commencer, afin de cartographier le douloureux périple de Fabien Didier Yene, d'énumérer les principaux faits qu'il relate dans son témoignage. Après un premier exil, initiatique en un sens, à N'Djamena au Tchad, l'auteur a d'abord survécu à son voyage périlleux en Afrique subsaharienne; bien qu'il « [se soit] fait dépouiller de tous ses sous » (Yene, 2010 : 99) par des policiers en traversant le Nigeria, il a réussi bon an mal an à poursuivre sa route au Niger, « le pays le plus pauvre qu'il ait connu jusquelà » (Yene, $2010: 101)$, et à atteindre la ville-carrefour d'Agadez, à la porte du désert. Il a survécu ensuite à la terrible traversée du désert du Ténéré jusqu'à Ghât en Libye " deux semaines dans des conditions [...] horribles » (Yene, $2010: 140$ ), irrespirables, et à la merci de trafiquants d'êtres humains qui ont alors un pouvoir souverain de vie et de mort sur les migrants. Découvrant que « la vie en Libye était un enfer» (Yene, 2010 : 151) pour les Africains subsahariens, il a alors décidé de passer en Algérie dans le but d'atteindre le Maroc. En Algérie, il a cependant survécu à de nouvelles épreuves dans le désert du Sahara, en particulier lorsqu'il a connu la déportation à Tin Zaouaten, dans la province de Tamanrasset près de la frontière du Mali. Parvenant malgré tout à gagner le nord et à traverser « la frontière algéro-marocaine » durant « l'hiver [2004] » (Yene, 2010 : 192), il a survécu enfin à quelque huit années de blocage au Maroc, dont quatre dans des conditions d'extrême dénuement et de harcèlement policier dans les forêts proches des enclaves: la forêt de Gourougou entre Nador et Melilla et celle de Bel Younech aux abords de Ceuta. Pendant ces années en forêt, comme l'auteur me l'a précisé par ailleurs, il a survécu spécialement à trente-deux tentatives infructueuses de passer clandestinement les frontières euro-africaines des enclaves espagnoles : vingttrois tentatives de passer les barrières et neuf tentatives à la nage, dont une dernière tentative collective meurtrière sur laquelle s'achève son livre, « dans la nuit du 25 au 26 septembre $2007 »($ Yene, $2010: 260)$.

Il faut aussitôt préciser les limites d'un tel résumé, cependant. Comme le notait justement Jean Norton Cru dans Témoins, «il ne suffit pas de relater un chapelet d'incidents et d'accidents dans leur matérialité pour faire sentir le réel " (Cru, 2006: 165). En l'occurrence, il ne serait pas impossible qu'on lise ce résumé comme la trame rocambolesque d'un roman d'aventures, c'est dire comme il peut induire en erreur. Or, si Migrant au pied du mur est un bon témoignage, c'est précisément parce que l'auteur s'est avisé que « [1]es faits ne parlent pas d'eux-mêmes » (Perec, 1992 : 93-94) et que son récit rétrospectif n'est donc pas un rapport de faits objectivés. On peut regretter le parti que Fabien Didier Yene a pris de rédiger son récit à la troisième personne, bien qu'il procède du souci, louable au demeurant, de ne pas se mettre en avant voire de se mettre à distance de soi-même; j'y reviens, l'auteur a parfois cédé à la tentation romanesque de substituer à son expérience celle d'un autre migrant auquel il souhaitait rendre hommage, de sorte que la valeur d'attestation du témoignage, pourtant essentielle à ses yeux, n'est pas irréprochable dans toutes ses parties ${ }^{7}$. Cependant, il a bien tenu à produire un document personnel dans lequel il restitue à notre intention la façon dont il a vécu les faits. Notamment, il a pris soin de relater sa migration dans sa chronologie de sorte que nous puissions l'accompagner en chemin, comme en situation 
d'observation participante, et que nous comprenions ainsi au fur et à mesure de l'action les motivations, les réflexions, les doutes, les craintes, les joies et les souffrances, les espoirs et les désespoirs de l'exilé.

7 Je ne m'attarderai pas ici sur les raisons qui ont poussé Fabien Didier Yene à s'exiler ; comme il me l'a confié, dans ce passage du premier chapitre intitulé «La famille au Cameroun ", l'auteur s'est inspiré de l'histoire d'un autre. Quoi qu'il en soit de cette histoire camerounaise, il ressort du troisième chapitre - «Le franchissement des frontières »- que c'est un Fabien Didier Yene fort innocent qui trouve refuge au Tchad. Le "chairman " du "ghetto " camerounais ${ }^{8}$ à N'Djamena lui trouve un "air trop villageois » (Yene, 2010: 81), tant il lui semble peu conscient des réalités du monde qui l'entoure. Or il importe de souligner dans ce sens que c'est l'inexpérience qui est au principe du voyage migratoire de l'auteur. Elle explique à la fois ses représentations en arrivant au Tchad et dont se moque le "chairman", et celles que conditionne son expérience tchadienne.

Comme pour beaucoup d'autres jeunes gens, l'Europe ne fait pas du tout partie de l'horizon de Fabien Didier Yene lorsqu'il arrive au Tchad'. Tandis que, selon le " chairman ", leurs compatriotes qui ont atteint N'Djamena " cherchent des moyens de continuer ", il songe seulement pour sa part à «cherch[er] un job» (Yene, 2010: 80). C'est donc en étant confronté à la misère d'un pays « plus pauvre » (Yene, $2010: 86$ ) que le Cameroun et au contact de compatriotes exilés, que l'auteur forme le projet de rejoindre l'Europe. Il apprend alors à nommer les maux dont il a souffert au Cameroun et qu'il découvre au Tchad en termes de pauvreté et d'injustice sociale. Corrélativement, il se convainc peu à peu à l'écoute des autres que la seule vraie alternative à ces maux est de "gagner l'Europe avec, pour escales, la Libye, l'Algérie ou encore le Maroc », sachant qu'en transit dans ces " pays plus développés » du Maghreb, on peut trouver " pas mal de boulot » (Yene, $2010: 84)$. Enfin, il intériorise son statut de migrant empêché de "voyag[er] par avion pour l'Europe» (Yene, 2010: 80); il découvre en particulier qu'en tant que migrant illégalisé, il a intérêt à circuler sans son passeport, qui devient "un moyen de blocage », et à lui substituer, moyennant corruption, un faux "papier du HCR" (Yene, 2010: 87) de façon à disposer d'une « nouvelle identité » (Yene, $2010: 89$ ).

Dans un sens, Fabien Didier Yene est alerté dès le départ, et il en fera l'amère épreuve au Maroc lorsqu'il sera débouté de sa première demande d'asile : quelles que soient ses raisons de s'exiler, il risque fort en tant que Camerounais d'être rangé dans la catégorie des « réfugiés d'ordre économique » qui ne sont « pas les bienvenus » (Yene, $2010: 259$ ). C'est pourquoi le faux papier du HCR le fait passer pour un "réfugié ivoirien » (Yene, 2010 : 97), eu égard à la crise provoquée en Côte d'Ivoire par la tentative de putsch militaire du 19 septembre $2002^{10}$. Mais il est néanmoins très loin d'imaginer les épreuves migratoires qui l'attendent. C'est précisément l'écart entre la réalité de ce qu'il va vivre et les idées fausses qu'il s'en faisait au départ qui éclaire son projet testimonial : parce que, rétrospectivement, une telle ignorance lui paraît proprement aberrante. 


\section{Migrer en Afrique subsaharienne : mobilité illégalisée et corruption généralisée}

Les violences dues à son statut de migrant illégalisé surgissent vite au cours de son voyage, pourtant. Dès le Nigeria, "voyageur clandestin " sous sa fausse identité de «réfugié ivoirien » (Yene, 2010: 95-96), Fabien Didier Yene subit une fouille en règle lors d'un contrôle de police puis un chantage qui l'oblige, pour préserver sa vie, à céder tout son argent. Tout au long de son voyage en Afrique subsaharienne, il fait ainsi l'épreuve dans sa chair que les policiers «trouvent leur compte en fouillant et dépouillant les voyageurs, peu importe leur passeport » (Yene, $2010: 117)$, et parfois de la manière la plus brutale. Ces violences policières se poursuivent même un peu au-delà d'Agadez, jusqu'à la fin du bitume : l'auteur raconte qu'après le départ d'Agadez, les exilés de son pick-up sont encore victimes deux fois de ce qu'en tant que témoin oculaire, Fabrizio Gatti finit par appeler la « razzia habituelle» (Gatti, 2010 : 73), dans Bilal. Sur la route des clandestins. Or ces violences menacent la vie des migrants, tantôt en elles-mêmes, tantôt du fait qu'elles ruinent le projet migratoire. Bien que " désespéré » (Yene, $2010: 101$ ) après avoir réchappé à la police nigériane, Fabien Didier Yene ne conçoit pas de faire machine arrière. C'est ce qui arrive parfois : parti du Cameroun une première fois et parvenu au Niger, Kingsley Kum Abang a pour sa part «rebroussé chemin au Nigeria, faute d'argent » (Abang, 2006 : 156). Quant à lui, Fabien Didier Yene juge qu'il « lui [est] impossible» de «rentrer au Tchad» (Yene, 2010 : 99), sans même parler du Cameroun où il "savait que son passé était un obstacle pour son avenir " (Yene, 2010:121). Quand on ne peut ni poursuivre sa route ni rentrer dans son pays, le risque est grand de sombrer. Dans sa prostration, l'exilé semble l'imaginer après avoir passé la frontière du Niger, mais ce n'est pas qu'une vue de l'esprit :

«On lui montra deux hommes arrivés depuis environ quatre mois. Ils avaient été dépouillés par les Nigérians avant d'arriver à Maradi. Leur corps semblait avoir subi d'importantes tortures. Ils auraient été violés, une nuit. La mort ne leur faisait plus peur, personne ne leur venait en aide. [...] Ils étaient devenus fous. Plus rien à faire pour eux, surtout au Niger, où la sécheresse et la famine sont les problèmes primordiaux de la population, qui souffre énormément. » (Yene, 2010 : 103)

11 Selon le reporter Fabrizio Gatti, le terme usité à Agadez pour désigner ces hommes bloqués en cours de migration au Niger et privés de tout, en perdition, est le mot anglais «stranded », qui "signifie échoué, enlisé, laissé en souffrance, dans le pétrin, en difficulté » (Gatti, 2010: 83). De fait, trouver de nouvelles ressources pour continuer malgré les razzias policières ne va pas du tout de soi, «surtout au Niger ». Parfois, des migrants finissent par devenir ce que Fabien Didier Yene appelle des «hommes d'affaires »11, par exemple cette personne grise qu'est le « chairman » d'un « ghetto ${ }^{12}$.

Ces réalités de la mobilité illégalisée en Afrique subsaharienne, Fabien Didier Yene ne les accepte pas. Face aux policiers, il tente constamment de faire respecter ses droits, de refuser l'humiliation. Mais de façon générale, le cas des migrants ne lui apparaît que comme un cas parmi d'autres, peut-être seulement plus exposé. À l'exception de sa rencontre avec un policier nigérien épatant, car convaincu que la police est faite «[p]our la sécurité des citoyens et leur bien» (Yene, 2010: 103), il vérifie au Nigeria puis au Niger que, comme le lui disait son hôte Jean-Louis à N'Djamena, «[t]ous les policiers sont des escrocs " (Yene, $2010: 87)$. La corruption endémique de la police, qui lui était déjà familière au Cameroun ${ }^{13}$, est ainsi analysée comme exemplaire de l'injustice qui règne en Afrique. C'est une situation générale où l'État n'est qu'une 
baudruche («L'État, c'est qui, petit ?... ») et où «[c]e sont les affaires qui donnent » (Yene, 2010 : 94), tant et si bien que "l'expression biblique "On prendra à celui qui n'a pas pour ajouter à celui qui a" s'est transformée en "Le riche prendra à celui qui n'a pas le minimum pour en avoir encore davantage" » et que "[1]e pauvre n'est pas un homme libre» (Yene, $2010: 80)^{14}$. Si dans ces conditions « les gars se lancent vers des horizons divers » (Yene, $2010: 79$ ), c'est que l'emporte le sentiment que l'on « ne p[eut] rien dire ni changer » (Yene, $2010: 95)$.

Faisant partie intégrante de l'injustice africaine que les migrants entreprennent de fuir, les violences policières sont en somme vécues et décrites par l'auteur comme un mal inévitable avant de pouvoir « dire au revoir à l'Afrique, à la pauvreté, à la misère, aux maladies, à l'injustice» (Yene, 2010: 126). La fausse conscience des policiers va d'ailleurs jusqu'à présenter le vol organisé de l'argent des migrants comme une sorte d'impôt perçu bien légitimement, puisque ceux-ci sont censés rejoindre bientôt l'eldorado ${ }^{15}$. Les migrants quant à eux sont avant tout «[d]es hommes et [d]es femmes qui cherchent la "liberté", jamais acquise dans leur pays d'origine", et qui rêvent de l'Europe comme d'un continent "de démocratie, de justice et $\mathrm{d}[\mathrm{e}]$ droits de l'homme " (Yene, 2010 : 145). Fabien Didier Yene témoigne cependant que, nourri par cet espoir de liberté et de justice, le soulagement de laisser enfin l'Afrique subsaharienne derrière soi est bien vite battu en brèche.

\section{D’Agadez à Ceuta : migration illégalisée et chosification}

Portant à la connaissance de tous l'expérience migratoire qui a été la sienne, Fabien Didier Yene espère transformer les consciences. La description qu'il fait des réalités vécues en Afrique subsaharienne dénonce l'idéologie qui préside à la caractérisation des "réfugiés d'ordre économique ", exclus du droit d'asile. L'auteur critique à cet égard la vision misérabiliste de l'Afrique, qui non seulement a le tort de substituer à l'analyse politique une optique humanitaire mais repose en outre sur des préjugés ${ }^{16}$. Quelle que soit la situation de pauvreté d'un pays à l'autre, l'intolérable pour ses compagnons de migration et lui, c'est avant tout de devoir vivre dans des pays qui, tel le Cameroun, sont "sans droit ni loi ", étant donné «les politiques des dirigeants africains» (Yene, 2010: 234). Dans le contexte d'une telle insécurité sociale, le problème spécifique que pose la migration illégalisée passe presque inaperçu. Même la collusion évidente entre les intérêts de la police et ceux des trafiquants d'hommes, à Agade $^{17}$, paraît dans l'ordre des choses. Cependant, la vérité éclate aux yeux de Fabien Didier Yene à compter de la traversée du désert nigérien - et de la façon la plus choquante.

Tandis qu'il pense se trouver enfin sur le chemin de la liberté, Fabien Didier Yene découvre en effet peu à peu la réalité de sa condition, qui est tout le contraire de la liberté. Comme le rappelle F. Gatti, la route que suivent les exilés dans le désert nigérien est anciennement la route des esclaves ${ }^{18}$; cette réminiscence historique est ce qui explique notamment que, dans le titre original de son livre, le reporter se dit infiltré «nel mercato dei nuovi schiavi » (dans le marché des nouveaux esclaves). De fait, ce que ses compagnons d'infortune et lui doivent subir à partir de ce moment, c'est ce que, bloqué au Maroc, Fabien Didier Yene appelle « chosification ${ }^{19}$. Ce terme désigne des violences aux multiples visages : celles des trafiquants d'hommes dans le désert; celles de citoyens du Maghreb, de la Libye au Maroc; celles des policiers et/ou des 
militaires des trois pays de transit ; celles, enfin, de la Guardia Civil espagnole. Mais il exprime avec acuité le dénominateur commun de ces violences, à savoir la négation des droits fondamentaux des migrants. Pointant une atteinte grave au droit international tel qu'il s'est élaboré progressivement depuis 1945, l'auteur accuse les politiques d'illégalisation des migrants d'être responsables de cette situation.

Le premier grand choc, pour Fabien Didier Yene, ce sont les traitements inhumains et dégradants que les trafiquants d'hommes réservent aux migrants durant la traversée du désert. Quand on est traités comme des marchandises, on ne peut qu'aller «de déception en déception, de surprise en surprise, d'escroquerie en escroquerie » (Yene, 2010 : 142). D'autant que, contrairement aux autres trafics, celui-ci ne contraint pas de livrer la « marchandise » à destination. Ainsi, malheur au migrant qui n'a plus d'argent avant la fin du voyage : il risque fort d'être abandonné dans le désert et livré à une mort presque certaine, malgré la somme qu'il a payée au départ. Fabien Didier Yene découvre ainsi un Camerounais et deux Nigérianes abandonnés en plein désert par leur chauffeur, « [f]aute d'argent » (Yene, $2010: 135)$. Inoussa, le Camerounais, explique :

« Notre chauffeur nous a bloqués pendant trois jours, puis, le quatrième, il nous a abandonnés ici au nombre de cinq. On voulait protester, il nous a montré les tas de sable qu'il y a devant le puits, en nous disant que c'était un convoi nigérian qui avait tapé sur le chauffeur, et [que] les Bozos [leur] ont réglé leur compte en les abandonnant la nuit ici. C'est la police nigérienne qui, en contrôlant le désert, les a trouvés morts et les a enterrés. » (Yene, $2010: 135)$

C'est ici la première appréhension, par Fabien Didier Yene, de la mort de masse des migrants qui tentent de gagner l'Union européenne. Les responsables politiques européens ne peuvent pas empêcher les mobilités des personnes qui aspirent à la liberté en leur interdisant les voies légales pour atteindre ce territoire. En revanche, ce faisant, ils font mine d'ignorer qu'ils les contraignent à emprunter des routes terriblement mortelles. On considère aujourd'hui à juste titre que «la frontière européenne en Méditerranée est devenue la route migratoire la plus dangereuse du monde " (Ritaine, 2015). Mais il convient d'analyser que cette "frontière européenne en Méditerranée " inclut le Sahara. Selon Fabien Didier Yene, les migrants qui parviennent au nord du Maroc ne s'y trompent pas: "quoique périlleuse », l'ultime étape matérialisée par les barrières des enclaves leur paraît « l'être moins que celle du désert qui avait fait périr de nombreuses personnes" (Yene, 2010: 208) ${ }^{20}$. Le récit d'Inoussa révèle en outre la cruauté de cette mort dans le désert, vouée à ne pas laisser de traces. Tel est le destin de celles et ceux dont on nie les droits fondamentaux : ils meurent comme s'ils n'avaient jamais existé, de sorte que « les familles de ces victimes ne sauront jamais ce qui est arrivé à leur fils, ni s'ils sont morts, ni comment » (Yene, $2010: 209)^{21}$.

Ce crime de la chosification, cependant, ne fait alors que commencer. De même qu'il a très vite brisé l'espoir des exilés après le départ d'Agadez, il annihile cet espoir à peine ressurgi après l'épreuve du désert - et jour après jour. Désormais, dans le témoignage de Fabien Didier Yene, les expressions de la déception, du désespoir, de l'angoisse, de la stupéfaction, s'enchaînent avec une telle récurrence que l'on se demande comment l'exilé a pu supporter tant de souffrance psychologique. Un signe stylistique ne trompe pas sur le contenu de celle-ci : c'est la multiplication en outre des comparaisons dégradantes des exilés à des animaux. Les exilés sont tantôt renvoyés ou repoussés " comme des chiens galeux " (Yene, 2010:151, 247), tantôt reçus « comme des chiens puants» (Yene, 2010: 215), tantôt «[1]igotés comme des chèvres qu'on mène à 
l'abattoir » (Yene, $2010: 256$ ) ; tantôt ils « viv[ent] dans les rochers ou au désert, comme des bêtes sauvages" (Yene, 2010: 184), tantôt ils sont réfugiés dans les forêts, où, " comme des rats-terriers", ils sont réduits à « se réveiller dans la nuit " pour «aller fouiller dans les poubelles près des habitations " (Yene, $2010: 223$ ).

Il est question ici de ce que, dans L'Espèce humaine, Robert Antelme caractérisait comme « la plaie du monde » (Antelme, 1997 : 56), à savoir le mépris. Au Maghreb, celui-ci se traduit notamment par des violences à caractère raciste. Le racisme se lisait sans nul doute déjà dans l'attitude des passeurs libyens, capables de se débarrasser des migrants dans le désert comme de déchets. Mais il devient vraiment manifeste pour Fabien Didier Yene à compter de son séjour en Libye, en particulier du fait des violences perpétrées par des personnes issues de la société civile. L'auteur dénonce ainsi exemplairement : la « rude exploitation » d'employeurs libyens prompts à «chosif[ier] de plus en plus les Noirs", vu que l'idée "prévaut [en Libye] que les Noirs sont plus serviles que l'âne ou le chameau » et qu'ils sont donc "corvéables à merci » (Yene, 2010 : 151) ; l'humiliation de chauffeurs de car algériens qui, quand ils ne refusent pas de le laisser monter à bord "[e]n raison de sa couleur de peau, comme s'il avait un aspect répugnant ", " asperg[ent] le car de désodorisant avant de démarrer " (Yene, 2010: 187-188); ou encore, au Maroc, les agressions commises par des «clochards barbares et racistes » qui « attaquaient les gars, leur broyaient les côtes, les violaient même sous la menace d'un couteau, avant de les livrer à la police » (Yene, $2010: 241$ ).

Cependant, il en va de ces "scènes de mépris, de racisme et de déshumanisation " (Yene, 2010 : 224) comme de celles perpétrées dans le désert: elles sont le fruit d'une politique. Dès lors que des responsables politiques traitent les migrants comme des indésirables, qu'ils les diabolisent en les désignant ouvertement pour cibles des violences policières et/ou militaires, il n'est malheureusement pas étonnant qu'une bonne partie de la population locale se montre résolument hostile à leur accueil. Fabien Didier Yene fait ressortir cette corrélation, lorsqu'il analyse à quel point le racisme est décomplexé au Maroc durant son séjour. Après avoir tenté sans succès de rafler les migrants près de la décharge publique de Nador, non loin de Melilla, la police marocaine a « donn[é] pour consigne aux clochards de les chasser de ces lieux, de les poursuivre, eux déjà affaiblis, envahis par la peur, la panique et l'angoisse ", de sorte que le jour même "tout le village " a commencé à « se manifest[er] par des scènes de xénophobie " (Yene, 2010: 223). Lorsque l'État marocain a "lan[cé] à nouveau une offensive contre les migrants clandestins en multipliant les rafles dans toutes les circonscriptions administratives », cela a «f [ait] les choux gras d'un journal populaire de la place qui titra un article: "Les criquets noirs porteurs de maladies ont envahi le royaume" "(Yene, $2010: 236)^{22}$.

21 Sans doute est-il juste de relier de telles réalités révoltantes à la situation générale en matière de démocratie et de droits de l'Homme, plus qu'inquiétante tant au Maroc qu'en Algérie et en Libye. Fabien Didier Yene raconte à plusieurs reprises comment, bafouant toutes les règles de droit international, l'État marocain organise des refoulements massifs de migrants. D'ordinaire, cela consiste à leur faire traverser illégalement et brutalement la frontière algérienne au nord, ce qui conduit parfois à un "ping-pong humain entre le Maroc et l'Algérie» (Mokhliss, 2008), les policiers algériens pouvant aussitôt ordonner aux migrants expulsés « de retourner du côté marocain, alors qu'on leur avait dit qu'ils seraient abattus s'ils tentaient de franchir de nouveau la frontière » (Yene, $2010: 208)$. Fabien Didier Yene témoigne de ce qu'il a ainsi 
" successivement subi huit refoulements " (Yene, $2010: 234)$ sans jamais se décourager de revenir aux abords des enclaves espagnoles. Quant au refoulement massif de migrants subsahariens dans le sud du désert marocain qui a fait suite à l'assaut massif de la barrière de Ceuta fin septembre 2005, l'auteur ne fait qu'en mentionner l'horreur en passant (Yene, $2010: 239)$ car il ne l'a pas vécue (lui-même ayant été alors refoulé à la frontière algérienne du côté d'Oujda); mais ce fait criminel responsable de nombreuses disparitions et qui est très documenté par ailleurs (voir Blanchard, Wender, 2007) en dit long lui aussi sur la négation des droits fondamentaux des migrants dont se rend coupable l'État marocain.

Toutefois, pour prendre la juste mesure de cette violence d'État qui loge les migrants subsahariens "à la même enseigne que les groupes terroristes " (Yene, 2010:226), il convient d'analyser avec Fabien Didier Yene qu'elle est intervenue, à l'époque où il $\mathrm{y} a$ séjourné malgré lui, dans le cadre d'accords négociés avec l'Union européenne et spécialement l'Espagne (voir Valluy, 2007 : 84-86). L'auteur ne cesse ainsi de mettre en lumière l'aide que les « gardes auxiliaires marocains » (Yene, $2010: 211$ ), les policiers et les militaires marocains apportent à la Guardia Civil espagnole dans son œuvre de blocage des exilés subsahariens. C'est là l'aspect le plus retors, car le plus souterrain, du "dispositif migratoire" mis en place par les responsables européens aux abords de leurs frontières extérieures. La militarisation de celles-ci passe par la construction de clôtures de fil barbelé concertina, doté de lames de rasoir sur lesquelles les migrants s'accrochent et se blessent (Yene, $2010: 212)^{23}$ mais aussi par cette "externalisation de l'asile » plus discrète, suivant laquelle la police et l'armée d'États tiers jouent le rôle de gendarmes de l'Europe. C'est ce qui explique, dans les forêts marocaines proches des enclaves espagnoles, les "rafles continuelles» dont les migrants éprouvent une «angoisse permanente» (Yene, 2010: 205) ainsi que les refoulements évoqués cidessus, qui font souvent suite à ces rafles.

La collaboration entre les forces de sécurité espagnoles et marocaines n'est jamais aussi manifeste que lorsque les migrants illégalisés se lancent collectivement à l'assaut de l'une des barrières ou qu'ils se risquent en mer pour passer la frontière à la nage ou en bateau pneumatique. Lorsqu'ils sont alertés d'un assaut, observe Fabien Didier Yene, les militaires marocains «suivent les instructions de la Guardia à la lettre»; ils poursuivent, arrêtent, enferment et brutalisent si bien les migrants «à coups de matraques ou de petits gourdins » qu'ils reçoivent en récompense « des cigarettes, du whisky ou encore des euros" (Yene, 2010: 204). Lorsque des migrants subsahariens sont tués, l'auteur accuse parfois des «balles marocaines» (Yene, 2010: 203) ou indifféremment « les tirs de balles des militaires marocains et espagnols» (Yene, 2010 : 239). Citant le journal qu'il a tenu en septembre 2007, il est en état d'attester le meurtre d'un Sénégalais rejeté à la mer par la Guardia Civil alors qu'il ne savait pas nager (Yene, 2010: 262-263). Mais ce qui ressort en général de cette externalisation de l'asile européenne, c'est une dilution des responsabilités telle que l'impunité règne.

C'est ce en quoi le témoignage de Fabien Didier Yene entre en résonance avec le film Les Messagers, auquel l'auteur a d'ailleurs apporté une contribution importante. Il fait apparaître qu'une logique criminelle est à l'œuvre, qui mène de l'illégalisation à la chosification - et finalement à la disparition anonyme dans la frontière, que celle-ci soit désertique ou maritime. Cachés, traqués, violentés, refoulés, assassinés, disparus, les migrants illégalisés sont de fait réduits à l'état de parias, d'êtres superflus. Dans ces conditions, témoigner en littérature et au cinéma de son expérience migratoire, c'est - 
comme chaque fois que l'on a commis un crime contre l'humanité dans l'histoire protester de son appartenance à l'espèce humaine une et indivisible. Ce n'est pas le faire théoriquement en invoquant un universel abstrait; mais c'est le faire pratiquement en réclamant que justice soit faite: que les faits criminels soient reconnus et qualifiés, que la chaîne des responsabilités soit établie - et qu'il résulte de cette connaissance, à l'avenir, une nouvelle effectivité du droit international.

\section{BIBLIOGRAPHIE}

Abang, Kingsley Kum ; Jobard, Olivier ; Saugues, Florence (2006) Kingsley. Carnet de route d'un immigrant clandestin, Paris, Marval, 155 p.

Améry, Jean ; Wuilmart, Françoise (trad.) (2005) Par-delà le crime et le châtiment. Essai pour surmonter l'insurmontable,Arles, Actes Sud, 209 p. (Babel).

Antelme, Robert (1997) L'Espèce humaine, Paris, Gallimard 306 p. (Tel).

Ballif, Florine ; Rosière, Stéphane (2009) Le défi des « teichopolitiques ». Analyser la fermeture contemporaine des territoires, L'Espace Géographique, vol. 38, pp. 193-206 [réf. du 08/08/2017] [Disponible sur internet].

Bigo, Didier ; Guild, Elspeth (2003) Schengen et la politique des visas, Cultures \& Conflits, $\mathrm{n}^{\circ} 49$ [réf. $\mathrm{du}$ 08/08/2017] [Disponible sur Internet].

Blanchard, Emmanuel ; Wender, Anne-Sophie (dir.) (2007) Guerre aux migrants. Le livre noir de Ceuta et Melilla, Paris, Éd. Syllepse, 234 p. (Arguments et mouvements).

Cayrol, Jean (1953) Témoignage et littérature, Esprit, vol. 21, n 4, pp. 575-578.

Crosby, Andrew ; Rea, Andrea (2016) La fabrique des indésirables : pratiques de contrôle aux frontières dans un aéroport européen, Cultures \& Conflits, $n^{\circ}$ 103-104, pp. 63-90.

Crouzillat, Hélène ; Tura, Laetitia [réal.] (2014) Les Messagers, Paris, Centre national de la cinématographie [éd., distrib.], DVD - 1h 10 min..

Cru, Jean Norton (2006) Témoins : Essai d'analyse et de critique des souvenirs de combattants édités en français de 1915 à 1928, Nancy, Presses universitaires de Nancy727 p. (Histoire contemporaine).

Dauvergne, Catherine (2008) Making People Illegal. What Globalization Means for Migration and Law, New York, Cambridge University Press, 216 p.

Detue, Frédérik (juin 2017) Le lieu et la parole. Les Messagers, documentaire lazaréen, La Revue Documentaires. Disparition(s), $\mathrm{n}^{\circ} 28$, pp. 24-33.

Feldman, Gregory (2011) The Migration Apparatus. Security, Labor, and Policymaking in the European Union, Stanford, Stanford University Press, 224 p.

Gatti, Fabrizio ; Defromont, Jean-Luc (2010) Bilal. Sur la route des clandestins, Paris, Liana Levi, 477 p. (Piccolo). 
Heller, Charles ; Pezzani, Lorenzo (2014) Traces liquides : enquête sur la mort de migrants dans la zone-frontière maritime de l'Union européenne, Revue européenne des migrations internationales, vol. 30, n³-4, pp. 71-107.

Kanstroom, Daniel ; Menjívar, Cecilia (dir.) (2014) Constructing Immigrant "Illegality”. Critiques, Experiences, and Responses, New York, Cambridge University Press, 399 p.

Levi, Primo ; Schruoffeneger, Martine (2003) Si c'est un homme, Paris, Pocket, 314 p. (Presses Pocket).

Mokhliss, Brahim (2008) Migrants subsahariens. Ce ping-pong humain entre le Maroc et l'Algérie, lereporter.ma [ref. du 24/09/2017] [Disponible sur internet]

Perec, Georges (1992) L.G. Une aventure des années soixante, Paris, Le Seuil, 183 p. (La Librairie du $\mathrm{XX}^{\mathrm{e}}$ siècle).

Ritaine, Évelyne (2015) Du pouvoir d'exposer à la mort à/par la frontière, Cultures \& Conflits $\mathrm{n}^{\circ}$ 99-100, [réf. du 05/05/2017] [Disponible sur Internet]

Traoré, Mahmoud ; Le Dantec, Bruno (2017) Partir et raconter : " Dem ak xabaar ». Une odyssée clandestine, Fécamp, Lignes, 316 p. (Lignes poche).

UNHCR (2017) Global Trends. Forced Displacement in 2016, Report, 72 p. [réf. du 22/08/2017]

[Disponible sur Internet].

UNICEF (2017) Enfants migrants d'Afrique. L'Europe n'est pas leur objectif premier selon l'UNICEF, unicef.fr [réf. du 12/08/2017] [Disponible sur Internet] (communiqué).

UNITED for Intercultural Action (2017) The Fatal Policies of Fortress Europe, unitedagainstracism.org [réf.du 15/08/2017] [Disponible sur Internet].

Valluy, Jérôme (2007) Les rafles de Subsahariens au Maroc, Vacarme, n 39, pp. 84-86 [réf. 25/09/2017] [Disponible sur Internet]

Yene, Fabien Didier (2010) Migrant au pied du mur, Biarritz-Paris, Atlantica-Séguier, 284 p.

\section{NOTES}

1. Le livre étant épuisé chez un éditeur qui a d'ailleurs cessé son activité, l'auteur a entrepris de retravailler son texte en vue d'une nouvelle édition.

Je remercie chaleureusement Fabien Didier Yene pour toutes les précisions qu'il m'a apportées. Je remercie également Laetitia Tura pour sa relecture bienveillante et attentive.

2. J'emploie ici un lexique adopté en sciences sociales par les chercheurs qui entendent "souligner l'effet des politiques publiques qui rendent l'immigration illégale » (Heller, Pezzani, 2014 : 72). Voir aussi, entre autres : Dauvergne, 2008 ; Kanstroom, Menjivar, 2014. Selon Elspeth Guild et Didier Bigo, l'un des «instruments privilégiés » d'une telle politique d'« irrégularisation » est le visa Schengen lui-même : «le visa ne facilite pas simplement la venue des étrangers dans l'Union, il est aussi et surtout un moyen de les tenir à distance et de mettre à l'écart les "indésirables" [...] en les empêchant de voyager» (Bigo, Guild, 2003).

3. Courriel que Fabien Didier Yene m'a adressé le 16.03.2016. Pour plus de précisions sur ces conditions, lire dans ce dossier l'entretien que l'auteur m'a accordé. Les imperfections du témoignage évoquées plus bas doivent être reçues à la lumière de ces conditions.

4. Dans son récit à la troisième personne, Fabien Didier Yene énumère ses activités militantes au Maroc à compter de 2006: "Il mit sur pied une association de Camerounais dite ADESCAM, Association de sensibilisation et de développement des Camerounais migrants au Maghreb 
(Maroc). Il fut membre actif du Réseau Manifeste euro-africain dénonçant les injustices dont sont victimes les migrants, et les effets néfastes des politiques dites sécuritaires, défensives ou protectionnistes. [...] En mars 2008, il fut élu président de la Communauté camerounaise des émigrés du Maroc, dans le but d'une médiation entre la grande famille migrante clandestine camerounaise et les autorités accréditées » (Yene, $2010: 260)$.

5. Le néologisme de «teichopolitique » a été forgé par les auteurs « sur la racine grecque teichos, "mur" (de la cité) » (Ballif, Rosière, 2009 : 193).

6. Sur le concept de «frontière-réseau ", voir Crosby, Rea, $2016: 66$.

7. C'est précisément un enjeu du travail de réécriture auquel se livre actuellement l'auteur que de corriger cette erreur, dans un récit à la première personne.

8. Voir : «À chaque étape stratégique du périple vers l'Europe, il y a des "ghettos", des camps de fortune où les voyageurs illégaux peuvent manger, se laver et se reposer avant l'escale suivante. Dans chacun de ces lieux d'hébergement, il faut payer son loyer et sa nourriture au chef, le “président". (Abang, $2006: 66$ ).

9. C'est ce qui ressort encore d'une étude commandée par l'Unicef et menée par Reach en 2017 ; voir UNICEF, 2017. Un autre rapport récent du HCR confirme la tendance selon laquelle, de façon générale, la grande majorité des requérants d'asile d'Afrique subsaharienne s'établissent dans un pays voisin du leur: "In sub-Saharan Africa, the vast majority of refugees remained in the immediately neighbouring countries »(UNHCR, $2017: 14)$.

10. En 2005 encore, au Maroc, les Ivoiriens se disent « des réfugiés [politiques], contrairement aux autres » (Yene, $2010: 244)$ et des Camerounais se font « passer pour des Ivoiriens » de façon à échapper à un rapatriement forcé (Yene, $2010: 249$ ).

11. Voir : "L'aventure se révélait être pour beaucoup une école de sagesse. On entendait même dire qu'un "jeune qui voyage est l'égal d'un vieillard au village". Nombreux sont ceux qui entrent dans le système "des hommes d'affaires". Le mal prend le dessus sur la raison, c'est une nouvelle façon de vivre pour tous ceux qui n'ont pas de moyens. Malgré les nombreux risques, la mentalité change, on raisonne avec cette idée que l'on a quitté son pays à la recherche d'argent. » (Yene, $2010: 171)$.

12. Comme l'expose Kingsley Kum Abang, le «chairman » du « ghetto » est « très souvent [...] un candidat à l'exil qui a essayé plusieurs passages sans succès. À court d'argent, il ne peut ni poursuivre ni rebrousser chemin. Alors, il fait des affaires pour gagner de quoi retenter sa chance : gestion du "ghetto". » (Abang, $2006: 66$ ).

13. «Au Cameroun, pour n'importe quelle interpellation, il faut donner de l'argent au policier. Ils appellent cela "payer la bière" ou "le droit du policier". » (Yene, $2010: 104)$.

14. Dans Si c'est un homme, Primo Levi soutient que c'est dans le Lager nazi d'Auschwitz que la loi féroce qui énonce "Il sera donné à celui qui possède, il sera pris à celui qui n'a rien » lui est apparue «ouvertement en vigueur et unanimement reconnue» (Levi, 2003 : 136). Cette «loi féroce » est énoncée dans le Nouveau Testament : «Car on donnera à celui qui a et il aura en plus ; mais celui qui n'a pas, on lui enlèvera même ce qu'il a » (Évangile selon Matthieu, XIII, 12).

15. Voir : « Vous venez chaque jour ici, à Agadez, de vos pays, pour aller en Europe. Vous pleurez quand vous partez, mais on n'a plus de vos nouvelles au retour [dans] vos pays. C'est comme cela que nous mangeons.» (Yene, 2010: 118). Voir également: "Ces fonctionnaires sous-payés pensent que nous sommes cousus d'or. Ils sont persuadés que nous dissimulons dans la couture de nos pantalons et dans nos slips les sommes faramineuses que réclament les passeurs successifs sur le chemin de l'Europe. Obligés de transiter par là [Agadez], les migrants sont K.-O. avant même d'affronter le désert. » (Traoré, $2017: 37$ ).

16. Voir : «Le problème, c'était de rencontrer des gens avec l'idée que tous les Subsahariens partaient de leur pays parce qu'ils n'avaient pas de quoi manger et que les guerres en Afrique étaient causées par la famine. Dieu merci, une grande partie de ce continent en est à l'abri. » (Yene, 2010 : 252). De fait, Fabien Didier Yene ne cesse d'observer les disparités de richesse entre 
les pays qu'il traverse, du Niger, qui lui paraît « le pays le plus pauvre qu'il ait connu jusque-là » (Yene, 2010 : 101), au Nigeria où il admire des infrastructures jamais vues au Cameroun (Yene, 2010 : 95-96), en passant par le Tchad, dont il découvre qu'il est quant à lui « plus pauvre » (Yene, 2010 : 86) que le Cameroun.

17. Voir : «Si tu payes le passeur et qu'ensuite il te plante là, tu n'as personne à qui te plaindre, tu es seul au monde - quand tu vas porter plainte, la police se moque de toi, elle est complice.» (Traoré, $2017: 43$ ).

18. Selon l'auteur, cette route du désert « fournissait déjà l'Empire romain en bras, il y a de cela deux mille ans. Encore actuellement, on la nomme la Piste des Esclaves. » (Gatti, 2010 : 87).

19. Dans la séquence finale du film Les Messagers d'Hélène Crouzillat et Laetitia Tura, Fabien Didier Yene explicite l'emploi qu'il fait du mot «chosification» (Crouzillat, Tura, 2014: 1 :00 : 46-1:03 :33). À propos de ce film, voir Detue, 2017.

20. Dans sa tâche de documenter les «morts de réfugiés et de migrants causées par les politiques restrictives de l'Europe forteresse ", le réseau associatif européen UNITED for Intercultural Action ne néglige pas d'inclure les morts survenues dans le désert (UNITED, 2017).

21. Le film Les Messagers, qui se consacre tout entier à la disparition des exilés dans la frontière, s'ouvre sur l'évocation d'un groupe de migrants qui s'est perdu dans le désert après deux jours de marche. Les réalisatrices montent une image photographique de paysage désertique avec une voix de témoin tout aussi fruste. Vient le moment, dans le récit de celui-ci, où le groupe s'arrête et décide d'attendre la mort. Leur dernière volonté, cependant, est de ne pas disparaître : « Ils ont pris leur carte d'identité. Ils l'ont accrochée sur eux comme ça, là, à un niveau où ça ne va pas tomber, même si le vent vient. » (Crouzillat, Tura, 2014 : 0 :00:20-0:01:05).

22. Inversement, observe Fabien Didier Yene: «Dans certains lieux loin des villes, l'imam semblait être le chef hiérarchique de la société, et on assistait à un respect et une soumission des populations lorsqu'il demandait qu'on accueille les migrants. » (Yene, $2010: 234$ )

23. Mahmoud Traoré se blesse gravement lors de l'assaut du 28 septembre 2005 : voir le récit glaçant qu'il fait de son expérience dans le chapitre "À l'assaut de la dernière frontière " (Traoré, 2017 : 249-261).

\section{INDEX}

Mots-clés : migration irrégulière, politique migratoire, accueil, témoignage, contrôle des frontières, littérature

Index géographique : Cameroun, Tchad, Nigeria, Niger, Sahara, Libye, Algérie, Maroc, Espagne, Melilla, Ceuta

\section{AUTEUR}

\section{FRÉDÉRIK DETUE}

Maître de conférences en littérature générale et comparée, Université de Poitiers - FoRRel (EA

3816), équipe B3

frederik.detue@univ-poitiers.fr 